\title{
Bounds on the Achievable Rates of Faded Dirty Paper Channel
}

\author{
Zouhair Al-qudah ${ }^{1}$, Wael Abu Shehab ${ }^{2}$ \\ ${ }^{1}$ Department of Communication Engineering, Al-Hussein Bin Talal University, Ma'an, \\ Jordan \\ ${ }^{2}$ Department of Electrical Engineering, Al-Hussein Bin Talal University, Ma'an, Jordan
}

\begin{abstract}
Bounds on the achievable rate of a Gaussian channel in the case that the transmitter knows the interference signal but not its fading coefficients are given. We generalize the analysis which were studied in many other references such that their results are special cases of our analysis. We enforce our bounds by simulations in which many numerical examples are drawn and investigated under different cases.
\end{abstract}

\section{KEYWORDS}

Dirty paper coding, lattice encoding and decoding, fading channel, Viterbi Algorithm.

\section{INTRODUCTION}

Dirty paper coding (DPC) is a communication model deals with Gaussian noise non-causally known at the transmitter. In this communication model, an encoding technique is used with such a noise such that its effects can be cancelled completely as Costa showed in his valued paper. The received signal, $Y$, is decribed as

$$
Y=X+S+Z
$$

where $X$ is the transmitted signal and its average power is constrained by $E\left[X^{2}\right] \leq P_{X}$. Further, $S$ is the interference non-causally known signal at the transmitter and with power constrained by $E\left[S^{2}\right] \leq P_{S}$. In addition, $Z$ is the additive white Gaussian noise vector (AWGN), with average power $P_{Z}$. DPC has a wide variety of applications includes MIMO broadcast channel where a perfect knowledge of the interference is available, information hiding and digital watermarking.

In [6], Costa showed that the capacity for this channel doesn't change when the interference is non-causally known at the transmitter, and later in [9], they generalize this result for an arbitrary interference distribution if the noise is Gaussian that is, 


$$
C=\frac{1}{2} \log _{2}\left(1+\frac{P_{X}}{P_{Z}}\right)
$$

In [1], the authors studied the channel model in the case that a lack of interference phase knowledge at the transmitter, that is $Y=X+e^{j e} S+Z$ where $\emptyset$ is quasi static and random. In [theorem (1),[1]], the authors derived an upper bound of the capacity when the phase $\emptyset$ takes one of the two values $\{0, \pi\}$. That is the transmitter does not know the interference in perfect, instead, it has to select between two points. In [7], they showed that in such a situation, the system is equivalent to a transmitter wants to send a message simultaneously to two receivers where each channel add a different interference. Also, the authors in [1] derived an upper bound on the outage capacity (corollary 1) when the interferer signal has constant magnitude fading.

In [4], the autors followed [1] and studied the compound channel formulation instead of outage probabilities. In particular, they derived lower and upper bounds on the achievable bounds when the interference phase is unknown at the transmitter. Their derivation based on an adaption of the nested lattice approach that is described in [5] for the classic non fading channel and in [3] for the case of magnitude fading.

In [2], the authors generalized Costa's formulation, by multiplying $X$ and $S$ by random fading coefficients $\beta_{1}$ and $\beta_{2}$, respectively, in which the realizations are known only to the receiver. In addition, they modelled the coefficients as quasi static and assumed that these coefficients are real valued Rician random variables. (The power inflation factor becomes zero when the fading coefficients have zero mean, so, they consider the Rician fading channel). They studied the achievable outage probabilities when the interference signal is known to the transmitter but not the fading coefficients.

In [3], the authors considered a similar formulation where $\beta_{1}$ was constant and $\beta_{2}$ was real valued and random. That is $Y=X+S / \beta+Z$ where $\beta \epsilon[1-\Delta, 1+\Delta]$. Further, a lower bound is given when the power inflation factor is deterministic and an upper bound is given when the power inflation factor at the transmitter is random under the following conditions $\Delta \leq 1 / 3$, $S I R \rightarrow 0, S N R \rightarrow \infty$ that is noise free channel, their analysis based on lattice based coding scheme. In their work, they showed that proactively injecting randomness into the transmission strategy can produce substantial benefits.

In this paper, we generalize the results of $[1,3,4]$ where we assume that the transmitter has lack of magnitude and phase knowledge. We refer to such a situation as the faded dirty paper channel(FDPC). In this case, the received signal can be modelled as follows

$$
Y=X+h S+Z
$$

where $h$ is a random quasi static fading parameter, and it is given by $h=\gamma e^{j \phi}$. The channel has to support different transmission rates depending on how the fading parameter $h$ varies, where $h$ can vary in magnitude or phase or both. We extend the lower bound derived in [4] and generalize the upper bound that were firstly proposed in [1] for a predetermined values of $\phi$ and then generalized to an arbitrary value of $\phi$ in [4]. 
The rest of this paper is organized as follows, we review the principles of lattice dirty paper coding in Section 2. Lower bounds and upper bounds are presented in Section 3. Bounds are simulated and discussed in Section 4. Finally, we conclude our paper in Section 5.

\section{LATTICE DirTy PAPER CODING: DeFINITIONS AND PRELIMINARIES}

Lattices are firstly used in [5] to achieve the capacity of dirty paper channel. Let $\Lambda$ be a kdimensional lattice with fundamental Voronoi region $v$ with average second moment $P(\Lambda)=P_{X}$ and normalized second moment $G(\Lambda)=\frac{P(\Lambda)}{V^{\frac{1}{k}}}$ where $V=\operatorname{Vol}(v)$, then the lattice transmission procedure is as follows, [5,8]. At first, the sender generates the signal $X=[c-\alpha S-d] \bmod \Lambda$ where the input alphabet $X$ is restricted to $v$. Further, the $\bmod \Lambda$ is defined as $u \bmod \Lambda=u-Q_{\Lambda}(u)$ where $Q_{\Lambda}(u)$ is the lattice vector that is nearest to $u$ inters of the Euclidean distance. At the receiver, the destination computes $Y^{\prime}=[\alpha Y+d] \bmod \Lambda$ such that the received signal is reduced to $Y^{\prime}=\left[c+Z^{\prime}\right] \bmod \Lambda$ where the total noise at the destination is described by $Z^{\prime}=[(1-\alpha) d+\alpha Z] \bmod \Lambda$.

\section{BOUNDS OF FADED DIRTY PAPER CHANNEL}

In this section, a lower bound and an upper bound on the achievable rates are derived when the transmitter knows the interference non-causally but not the fading coefficients, that is the channel adds faded interference.

\subsection{Lower Bound}

We follow [4] in which the signal has a block of $n$ bits long. Then, each block is divided into $k$ blocks where every block contains $n / k$ bits. A $k$ dimensional lattice is used to construct the transmitted signal at each time block, the lattice is defined over complex number field. The encoding and decoding procedures are as follows. In this procedure, by using modulo lattice channel encoding scheme, the phase faded AWGN noise channel is converted such that it contains only the transmitted codeword and the total noise over this channel. In this case, the transmitted signal is given by

$$
x_{i}=\left[c_{i}-\alpha_{i} s_{i}-d_{i}\right] \bmod \Lambda
$$

for $i, i=1, \cdots, n / k$. In addition, $c_{i}, s_{i}$ and $d_{i}$ are the symbols of codeword covers the intended signal, interference signal and the dither signal at time $i$, respectively. The minimization factor $\alpha$ can be selected randomly as in [4] or to be selected as $\alpha=P_{X} /\left(P_{X}+P_{Z}\right)[3,5,8]$. This is because the encoder does not have knowledge of the realizations $h$ and $\phi$. The destination computes $y_{i}^{\prime}=\left[\beta_{i} y_{i}+d_{i}\right] \bmod \Lambda$ where $\beta_{i}$ is the noise minimization factor computed at the receiver and differs than $\alpha_{i}$ and it will be determined later. Here, $y_{i}^{\prime}$ is evaluated as follows: 


$$
\begin{aligned}
y_{i}^{\prime} & =\left[\beta_{i} y_{i}+d_{i}\right] \bmod \Lambda \\
& =\left[\beta_{i} x_{i}+\beta_{i} \gamma e^{j \phi}+\beta_{i} z_{i}+d_{i}\right] \bmod \Lambda \\
= & {\left[\beta_{i} x_{i}+c_{i}-x_{i}-d_{i}+\beta_{i} \gamma e^{j \phi}+\beta_{i} z_{i}+d_{i}-\alpha s_{i}\right] \bmod \Lambda } \\
& =\left[c_{i}-\left(1-\beta_{i}\right) x_{i}+\left(\beta_{i} \gamma e^{j \phi}-\alpha\right) s_{i}+\beta_{i} z_{i}\right] \bmod \Lambda
\end{aligned}
$$

In (5), the received signal contains i) the transmitted codeword $c_{i}$ at time $i$, and ii) the total noise $z_{i}=-\left(1-\beta_{i}\right) x_{i}+\left(\beta_{i} \gamma e^{j \phi}-\alpha\right) s_{i}+\beta_{i} z_{i}$. This total noise is independent of $c_{i}$. In addition, the components $x_{i}, s_{i}$, and $z_{i}$ are assumed to be independent of $c_{i}$ due to the dither signal. So, for a given values of $\alpha, \gamma$ and $\phi$, the variance of the total noise is given by

$$
P_{Z}^{\prime}=\left(\left|1-\beta_{i}\right|^{2} P_{X}+\left|\beta_{i} \gamma e^{j \phi}-\alpha\right|^{2} P_{S}+|\beta|^{2} P_{Z}\right)
$$

where $\beta_{i}$ is selected to minimize the variance of the effective noise. Then, $P_{Z}^{\prime}$ is minimized by selecting $\beta_{i}$ as

$$
\beta_{i}=\frac{P_{X}+\gamma \alpha e^{-j \phi} P_{S}}{P_{X}+\gamma^{2} P_{S}+P_{Z}}
$$

We denote $P_{Z}^{\prime}(\alpha, \beta, \gamma, \phi)$ the value of $P_{Z}^{\prime}$ in (6) computed at the value of $\beta_{i}$ from (7). The lower bound on the capacity for such a construction is given in [4] for phase faded dirty paper and we extend to faded dirty paper channel. This lower bound is given by

$$
R_{L B}(\alpha, \beta, \gamma, \phi)=E_{\alpha}\left\{\left[\log _{2}\left(\frac{P_{X}}{P_{Z}^{\prime}(\alpha, \beta, \gamma, \phi)}\right)-\log _{2}(\pi e G(\Lambda))\right]^{+}\right\}
$$

Where $\alpha$ is selected randomly with distribution known to the encoder and for any real value $x$, where $[x]^{+}=\max (0, x)$. A similar formulae was given in [3] for magnitude faded interference when the value of $\alpha$ is constant and equal to $P_{X} /\left(P_{X}+P_{Z}\right)$. A lower bound $G(\Lambda) \geq(1 / \pi e)$ was shown in $[12,13]$. This means that the second term in (11) is almost negative and its maximum value is zero.

\section{Remarks (1):}

1. We note that the formulae in (10) can be reduced to that given in [4] in the case of $\gamma=1$ for phase faded. In addition, the formulae in (10) can be reduced to that given in [3] for magnitude fading and $\phi=0$ or to the case that the channel adds a perfect interference without fading, that is $\alpha=\beta$. 
2. In the case of strong interference, the minimization factor is selected to cancel the interference effects, that is $\beta=(\alpha / \gamma) e^{-j \phi}$, and so the effective noise is reduced to $P_{Z}^{\prime}=\left(\left|1-\beta_{i}\right|^{2} P_{X}+|\beta|^{2} P_{Z}\right)$.

\subsection{Upper Bounds}

We now analyze the achievable upper bound over faded dirty paper channel. Our bound is valid for arbitrary $h$, where $h=\gamma e^{-j \phi}$ can vary in magnitude and phase. Further, we generalize theorem $(1,[4])$ which was given for phase faded dirty paper channel. Our bound is given in the following lemma

Lemma1: The capacity of the compound faded dirty paper channel is upper bounded by

$$
R_{U B}\left(\phi_{1}, \phi_{2}, \gamma_{1}, \gamma_{2}\right)=\left\{\begin{array}{lr}
\frac{1}{2} \log _{2}\left(1+\frac{P_{X}}{P_{Z}}\right) & P_{S}>v \\
\min \left(J\left(\phi_{2}-\phi_{1}, \gamma_{1}, \gamma_{2}, P_{X}, P_{S}, P_{Z}\right), \frac{1}{2} \log _{2}\left(1+\frac{P_{X}}{P_{Z}}\right)\right) & P_{S} \leq v
\end{array}\right.
$$

where

$$
J\left(\phi_{2}-\phi_{1}, \gamma_{1}, \gamma_{2}, P_{X}, P_{S}, P_{Z}\right)=\frac{1}{2} \log _{2}\left(\frac{\left(P_{X}+\frac{\gamma_{1}^{2}+\gamma_{2}^{2}}{2} P_{S}+P_{Z}+2\left|\cos \left(\frac{\phi_{2}-\phi_{1}}{2}\right) \sqrt{P_{X} P_{S}}\right|\right)^{2}}{P_{Z} P_{S}\left(\gamma_{1}^{2}+\gamma_{2}^{2}-2 \gamma_{1} \gamma_{2} \cos \left(\phi_{2}-\phi_{1}\right)\right)}\right)
$$

This bound is defined over arbitrary fading parameters $\gamma_{i}$ and $\phi_{i}$. In addition, this bound is achieved by time sharing for the values of interference power such as $P_{S}>v$, where $v$ is a parameter determined so that the bound is not an ascending function of $P_{S}$. Also, the upper bound can't be larger than the AWGN channel capacity. The proof of this lemma is a direct generalization of ( Theorem1, [4] ) and is omitted.

Remarks (2):

1. In the case that $\gamma_{1}=\gamma_{2}=1$, then, the formula in [4], which was derived for phase faded dirty paper channel, is obtained.

2. In the case that $\gamma_{1}=\gamma_{2}=1$, and $\phi_{2}-\phi_{1}=180^{\circ}$, then, we obtain $\operatorname{Eq}(8)$ in [1].

\section{Simulations AND DiscuSSIONS}


We start our discussion by comparing the upper bound and lower bound for faded (magnitude, phase, or combined) dirty paper channel. The following values $P_{X}=12 \mathrm{~dB}$ and $P_{Z}=0 \mathrm{~dB}$ are used. In addition, the bounds are plotted as a function of $P_{S}$.

Figure.1 compares among magnitude, phase and combined of both for a choice of $\left(\phi_{1}=0, \phi_{2}=\pi\right)$ and $\gamma_{1}=\gamma_{2}=1.2$. It shows that for values of $P_{S} \leq 9 d B$, the achievable rate of the phase faded performs better than faded (magnitude and phase). For values of $P_{S}>9 \mathrm{~dB}$, both bounds are achieved by time sharing, that is the channel capacity is achieved by time sharing . We note also that the upper and lower bounds for magnitude fading coincide (for range under consideration) and achieved by the time sharing. For lower bound with constant $\alpha$, it was found to be calculated as $\hat{\alpha} e^{j \phi_{0}}$ [4], where $\hat{\alpha}$ is determined by exhaustive search over a fine grid. Further, $\phi_{0}$ is set to the mean of $\phi_{1}$ and $\phi_{2}$. In addition $\gamma$ is set to the mean of $\gamma_{1}$ and $\gamma_{2}$. For the lower bound with variable $\alpha, \phi_{0}$ has a distribution that is independent of $\phi$. In addition, this figure shows that for low values of $P_{S}$, lower bound with constant $\alpha$ doing better than variable $\alpha$. These bounds coincide when $P_{S}$ increases and finally they reach their final values where the lower bound is achieved by time sharing.

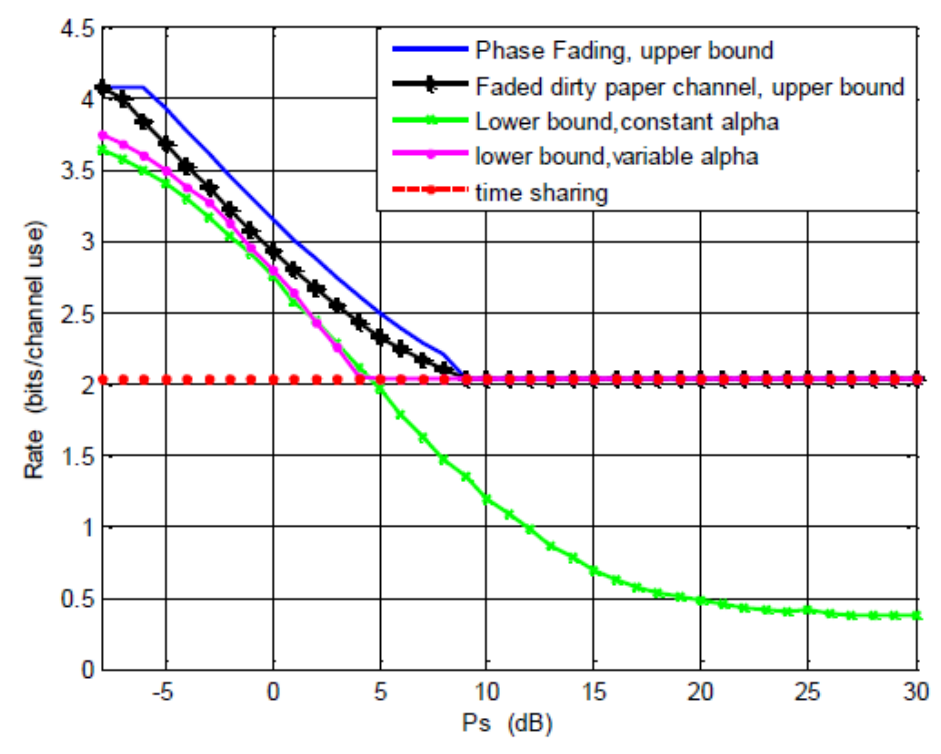

Figure 1. Bounds for dirty paper channel.

In an another example, Figure. 2 shows the same bounds when the magnitude fading parameters are changed to $\gamma_{1}=1.2$ and $\gamma_{2}=2$. It shows that the gap between the upper bounds for phase faded and faded interference is larger than the previous case due magnitude fading parameter. It shows also that the gap between the lower and upper bounds is slightly less than the previous case. Lower bound with variable $\alpha$ is better than with constant $\alpha$. Again for values of $P_{S}>9 d B$, the bounds can coincide and the channel capacity is achieved by time sharing. Again 
for only magnitude fading, the bounds coincide and are achieved by time sharing and so the channel capacity.

Figure. 3 shows the same bounds for $\left(\phi_{1}=0, \phi_{2}=\frac{2}{3} \pi\right)$, and $\gamma_{1}=\gamma_{2}=2$. It shows that the gap between the upper bounds with phase faded and faded dirty paper channel larger than the previous cases due to increasing the magnitude of the fading parameter. Lower bound is achieved by time sharing for values of $P_{S}>0 d B$ while the upper bound is higher. Discussion of lower bounds as in the first case just the point of achieving this bound by time sharing is changing from a case to another as we mentioned in section III.B .

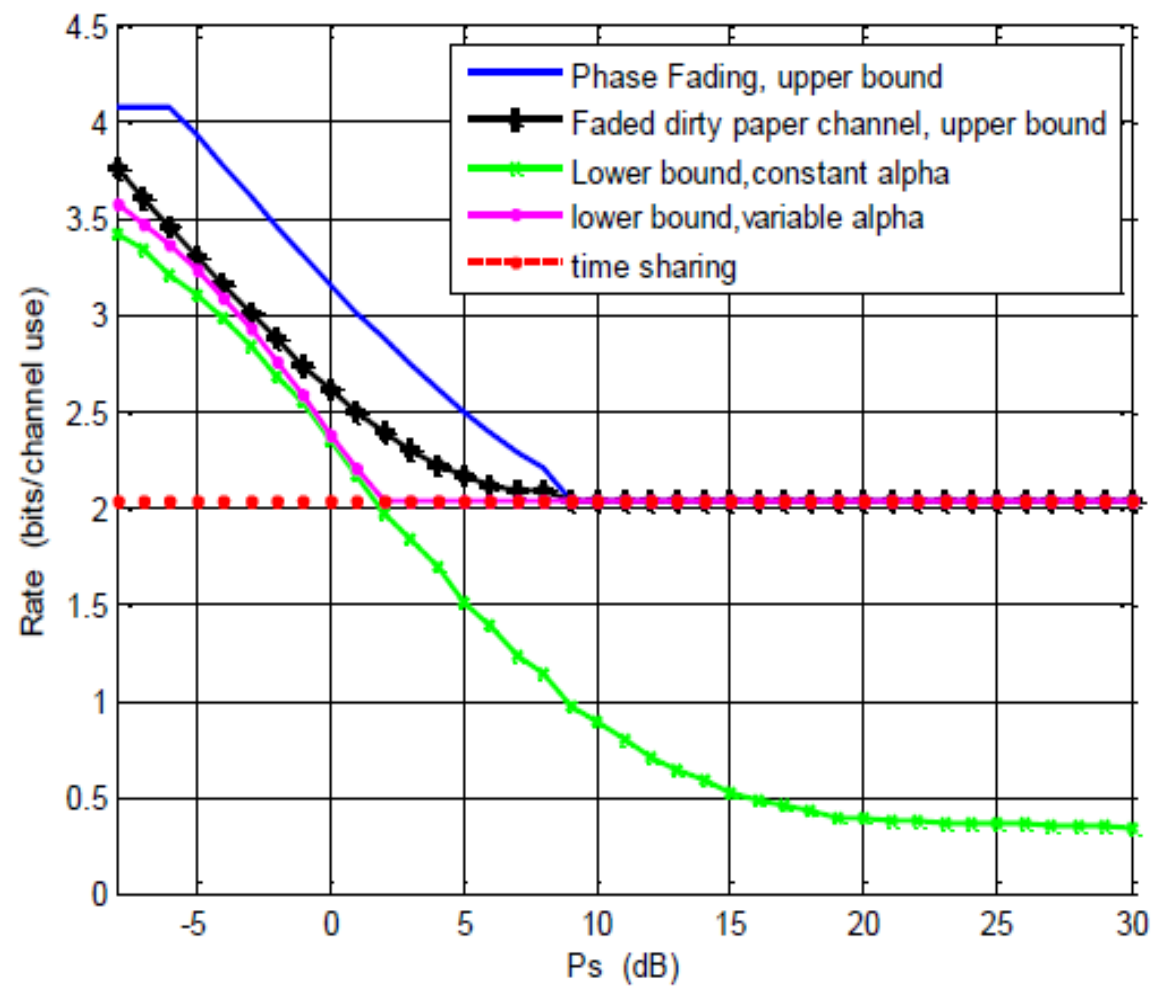

Figure 2. Bounds for dirty paper channel. 


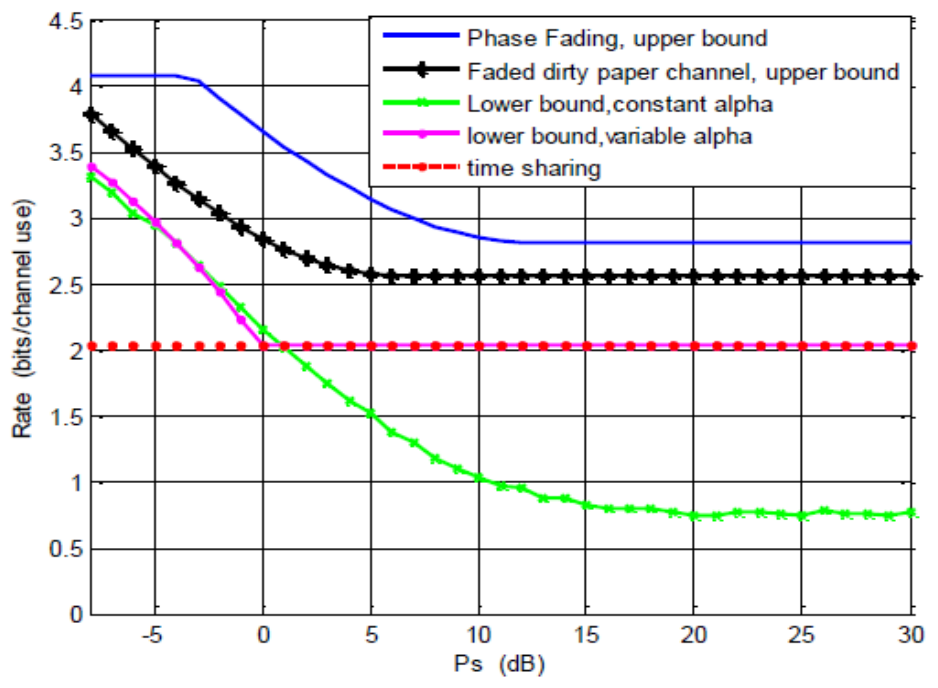

\section{Conclusions}

Figure 3. Bounds for dirty paper channel.

The faded dirty paper channels with arbitrary coefficients have been studied. The results in [1] and [4] have been generalized. We show our bounds by simulations for different cases. We have studied also the performance of faded dirty paper channel and we have showed how the system performance changed when the interference is faded.

\section{REFERENCES}

[1] P. Grover and A. Sahai,(2007) "What is needed to exploit knowledge of primary transmissions?," available at arXiv:cs/0702071.

[2] P.Mitran,N.Devroye and V.Tarokh, (2006) " On Compound Channels with Side Information at the Transmitter”, IEEE Trans. on Info. Theory, vol. 52, no. 4, pp. 1745-1755.

[3] A.Khina and U.Erez, (2010) "On the Robustness of Dirty Paper Coding”, IEEE Trans. on Comm., vol. 58, no. 5, pp. 1437-1446.

[4] A.Bennatan,V.Aggarwal,Y.Wu,A.R.Calderbank,J.Hoydis ,and A.Chindapol, (2007) "Bounds and Lattice-Based Transmission Strategies for the Phase-Faded Dirty-Paper Channel”, IEEE Trans. on Wireless Comm., vol. 8, no. 7, pp. 3620-3627.

[5] U. Erez, S. Shamai and R. Zamir, (2005) "Capacity and Lattice-Strategies for Cancelling Known Interference," IEEE Trans. on Inform. Theory, vol. 51, no. 11, pp. 3820-3833.

[6] M.Costa, (1983) "Writing on Dirty Paper",IEEE Trans. On Information theory,vol 29, no.3,pp 439441.

[7] A.Cohen and A.Lapidoth, (2002) "The Gaussian watermarking game”,IEEE Trans. Info. Theory vol.48, pp.1639-1667.

[8] A.Cohen and A.Lapidoth, (2002) "The Gaussian watermarking game”,IEEE Trans. Info. Theory vol.48,pp.1639-1667

[9] U. Erez, S. Shamai (Shitz), and R. Zamir,(2005) "Capacity and lattice strategies for cancelling known interference," IEEE Trans. Information. Theory, vol. 51, pp. 3820-3833.

[10] G.D.Forney, (1988) “Coset Codes-Part I:Introduction and Geometrical Clasifications”, IEEE Trans. On Information Theory, vol. 34, no. 5, pp. 1123-1151

[11] R. Zamir, (2009) "Lattices are Everywhere", Information Theory and Applications (ITA09), University of California at San Diego, pp. 392-421. 
International Journal of Computer Networks \& Communications (IJCNC) Vol.9, No.1, January 2017

[12] R.Zamir,S.Shamai and U.Erez, (2002) "Nested Linear /Lattice codes for structured multidimensional binning",IEEE Trans. On Information Theory, vol. 48, no. 6, pp. 1250-1276.

[13] R.Zamir and M.Ferder, (1996) "On lattice quantization noise “, IEEE Trans. On Information Theory,vol 42,no.4,pp1152-1159.

[14] U.Erez and S.t,Brink, (2005) "A close-to-capacity Dirty Paper Coding Scheme”, IEEE Trans. on Information Theory, vol. 51, no. 10, pp. 3417-3432.

[15] M.Mzaaotti and M.Chiani, (2006)“A simple rate $1 / 2$ co-decoding scheme for Writing on Dirty Paper”, IEEE International Conference on Communications, Istanbul, pp. 1622-1627.

[16] G.B.Kyung and C. Wang, (2009) "On the Design and Challenges of Practical Binary Dirty Paper Coding", on the proceeding of 2009 IEEE Wireless Communications and Networking Conference, Budapest, pp. 1-6..

[17] J.Chen and M.Fossorier, (2002)"Near optimum Universal Belief propogation Based Decoding of Low-Density Parity Check Codes”, IEEE Trans. on Communications, vol. 50, no. 3, pp. 406-414. 